\title{
The Natural History of a Federally Funded Researcher
}

\author{
Jordan R. Green, Ph.D.
}

Associate Professor

Corwin Moore Chair in Communication Disorders

Department of Special Education and Communication Disorders

College of Education and Human Sciences

University of Nebraska-Lincoln

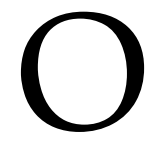

ver the past decade, many teaching universities have been aggressively growing their research programs. This swell of interest in academic research has occurred during a time when support from state funding sources for academic programs has declined. Consequently, the success of many universities as a whole has become highly dependent on the success of their research faculty. In this talk, I consider the growing scope of discipline of Communication Sciences and Disorders (CSD), current impediments to future progress in the field, and by way of example, the efforts of our federally-funded lab to address current research challenges. My perspective is that of an academic researcher whose career path has been supported continuously by grants from the National Institute on Deafness and Other Communication Disorders (NIDCD) starting from doctoral training to the obtainment of a large grant award. Also, highlighted are some examples of programs currently in place to promote research that have been implemented by NIH-NIDCD, the University of Nebraska-Lincoln (UNL), and the professional organization that supports my field, The American Speech-Language and Hearing Association (ASHA).

\section{Introduction to Communication Science and Disorders}

An estimated one in six individuals will suffer from a significant communication impairment in their lifetime. The ability to communicate is one of the most important human functions. Communication defines who we are - our personality, our relationships, and provides us access to the world. In some respects, my colleagues in the physical sciences are envious of the fact that I don't have to work very hard to convince others of the importance of research on human communication and its impairments. Also, that I don't have to search for interested funding agencies because $\mathrm{NIH}$ has an institute, NIDCD, that is dedicated primarily to funding research on communication disorders.

The profession of communication science and disorders (CSD) is a broad one, going beyond just speech and 
language, but also including audiology, cognition, swallowing, augmentative and alternative communication, professional voice, and even foreign accent reduction. The increasing scope of our profession poses a continual challenge with regard to training the next generation of practitioners and researchers. Speech science is a subdiscipline of CSD that encompasses the study of the anatomic, physiologic, neurologic basis of speech production and perception, and of the cognitive processes involved in communication. Speech science is supported by other subdisciplines including speechlanguage pathology and linguistics, anatomy and physiology, mechanical engineering, biomechanics, psychology, neuroscience, computer science, robotics, and genetics. The wide range of topics encompassed by CSD underscores the broad-level of expertise required to perform research on human communication and the necessity of interdisciplinary research.

In the United States, the field of CSD officially began in 1928. During that year, Sara Stinchfield was the first person in America to receive a Ph.D. degree in speech pathology and Raymond Stetson published an influential book called Motor Phonetics: A Study of Speech Movements in Action. Despite gradual progress, however, the pace of scientific discovery in the field of CSD is accumulating very slowly. The bases for most speech problems remain unknown and the diagnoses and prognoses of speech disorders are very subjective. Identifying objective criteria for classifying speech disorders has been an ongoing challenge and a major focus in the field.

\section{Impediments to Future Progress in CSD}

Progress in research has been slowed by a leadership crisis, methodological limitations, and federal funding shortages. Of course, many of these challenges are not unique to the discipline of CSD.

Leadership crisis: Perhaps our greatest challenge to preparing the next generation of leaders in CSD is in the recruitment of the next generation of scholars. The field of CSD currently faces a shortage of Ph.D. students with the number of graduates falling short of the number needed to replace retiring faculty. In 2001-2002, there were 333 unfilled slots for Ph.D. students according to a report (http://www.asha.org/students/academic /doctoral/phd_survey_sum.htm)

prepared by the Joint Ad Hoc Committee on the Shortage of Ph.D. Students and Faculty in CSD. Recruitment should begin at the undergraduate level. Unfortunately, only a small number of undergraduate and graduate students in our field are exposed to research. One very successful program that engages undergraduates in research at UNL is the Undergraduate Creative Activities and Research Experience (UCARE) program. This program is funded by the Office of Undergraduate Studies and funded by the Pepsi Endowment and Program of Excellence funds. The UCARE program provides undergraduates with funds to support a two-year research experience. More programs like UCARE are needed 
to raise students' awareness and interest in research careers.

High priority should be given to programs that facilitate the development of beginning investigators. A number of initiatives have been implemented by ASHA, NIH, and UNL to encourage the development of new investigators. NIH's programs include the Small Grant Program (R03) and High Program Priority (HPP). Post doc training also should be strongly encouraged. Unfortunately, post doc training is rare in the field of CSD because many of our Ph.D. graduates are being hired upon completion of their degrees or even before graduating. Without solid academic skills and a strong publication record, these individuals may be placed in a compromising position when seeking tenure at R1 institutions.

Another significant challenge to training the next generation of researchers is the shrinking supply of available mentors, which is partially due to the large number of impending retirements in our departments across the country. The mentoring of junior faculty needs to be a high priority as it maximizes the likelihood of success and improves retention by imbuing a sense of institutional loyalty in faculty who are frequently courted by other institutions. A working definition of mentoring needs to be articulated that is specific and institutionally endorsed. Tangible incentives should be provided for mentors and mentoring should be considered as part of academic load. Several UNL initiatives are in place to encourage the formation of mentored relationships between senior faculty, new faculty, and graduate students.
These initiatives are the Grant Mentor and the Preparing Future Faculty Programs.

The field is significantly shaped by decisions made by individuals serving as grant and manuscript reviewers. Poorly conceived reviews can be devastating for a new investigator. Yet, individuals who serve on these panels rarely undergo formal training and rarely receive feedback from peers or the institutions they serve. More programs like the NIH grant review training workshop sponsored by ASHA's Research and Scientific Affairs Committee - are needed to train the next generation of study section members and journal reviewers. This workshop provides beginning reviewers the opportunity to co-review grants with more seasoned reviewers who have served on standing NIH review panels.

\section{Methodological Limitations:} Overcoming methodological limitations is central to any successful research enterprise. The study of speech is no exception. Despite its obvious significance to quality of life, speech motor control is a topic that has been understudied when compared to other aspects of motor control in humans1. The dearth of scientific work is, in part, because speech is exceedingly difficult to measure. Speech is the result of activity of approximately 70 muscles and many parts of the speech production system, such as the tongue, soft palate and pharynx, are inside the body and therefore difficult to observe directly. Speech movements are also very small and rapid; when speaking, we produce up to 12 sounds per second. Therefore, new technologies are needed for 
accelerating the rate at which researchers can acquire and analyze speech data. Recognizing the general need for continued technological advancement, NIH offers the Shared Instrumentation Grant (S10) that provides up to $\$ 500,000$ annually for equipment related expenses needed by groups of NIH-funded investigators. These grants are important for providing researchers access to cutting edge technologies.

Continuing education is also needed to make researchers aware of emerging technologies. To assist in educating the scientific community, NIH-NIDCD has sponsored several state-of-the-science workshops. Another potential mechanism for continuing education includes the development of high quality E-learning on demand courses, particularly for continued education regarding robust and efficient experimental design and analysis techniques.

Federal funding shortages: Most of the speakers at this conference have commented on the challenges of maintaining and growing academic research programs given the current federal funding climate. Winning grants has become significantly more difficult for three primary reasons: (1) the growth rate of federal dollars for research has slowed, (2) the average grant size has grown, and (3) more researchers are competing for a smaller number of available grants. For example, in 1998 there were 24,000 NIH grant applications whereas in 2007, the number of applications were projected to be 49,000 (Giles \& Wadman, 2006). This reduction in the relative number of grants awarded annually will have deleterious effects on research and education in this country. The underfunding of research will also considerably weaken research training programs with fewer students exposed to research and fewer departments awarding doctoral degrees.

One necessary strategy to reduce the impact of the current funding crunch will be the formation of multicampus consortiums. Such alliances have numerous advantages. Consortiums accelerate pace of discoveries by providing the most efficient means for scientist with diverse perspectives to achieve scientific consensus. They also allow for comprehensive, multilevel level description of a large number of subjects, which is needed to obtain statistically sound descriptions of disorders. They also minimize variability across studies due to heterogeneity among subjects and differences across labs in data collection techniques.

\section{Efforts of one federally-funded lab to address challenges}

The long-term goal of our research program is to advance the scientific understanding of speech production and its disorders. To achieve this goal, we are investigating three aspects of speech production: (1) typical oromotor development for speech and feeding, (2) physiologic basis of impaired speech, and (3) computer analysis of speech motor performance. The brief discussion below focuses on our established research program on speech motor development and our emerging research program on amyotrophic lateral 
sclerosis (ALS). One principle that guides our research is that basic knowledge about speech motor development and typical speech performance is essential for improved differential diagnosis and treatment of speech motor impairments. Because the number of physiologic studies on speech production is fairly limited, one necessary and particularly challenging aspect of our work has been the development of new tools for recording, measuring, and analyzing speech performance. Our research program is student focused, with students of all levels working on research. This work would not have been possible without continued support from NIH-NIDCD.

As alluded to previously, a major impediment to progress in our understanding of speech motor development has been the lack of viable experimental methods for recording articulatory behaviors from very young children. To address this challenge, we have adapted 3D motion capture technology to obtain fine-grained measurements of face movements from infants and young children. The data from these studies are being used to address long-standing theoretical and practical issues regarding early speech development. The large amount of data now afforded by this technology, however, has also led to new challenges associated with data reduction and analysis. To address this challenge we have developed a software program (SMASH) to improve the efficiency and reliability of physiologic data reduction. In SMASH, multiple data files are loaded into a graphic user interface, custom analysis routines are accessible from dropdown menus, most routines rely on algorithmic detection, and measures are automatically exported to a database.

ALS, which is another focus of our research program on speech production, is a progressive neurodegenerative disease that affects motor nerve cells in the brain and the spinal cord. The mechanisms that trigger motor neuron death are unknown and no definitive biomarker for ALS has been identified. Life expectancy is only 2-5 years after diagnosis. The diagnosis of the disease is provisional based on clinical observations and neurologic symptoms in the absence of other causes. Because the clinicopathologic markers of ALS are so poorly defined, diagnostic uncertainty is very high; patients are initially misdiagnosed up to $45 \%$ of the time and the diagnosis can take up to 12 months. Early detection is becoming increasingly important with the number of experimental treatments growing rapidly in recent years; the benefits of drug therapies will be maximized if they are administered early in the disease while motor neurons are still intact. The goals of our research on ALS are to identify sensitive, quantitative indicators of disease progression that can be used as outcome measures for determining the effectiveness of experimental drugs and for improving the detection of disease onset and the prediction accuracy of disease progression in bulbar ALS. To achieve these goals, we are using the technologies we have developed to study early speech motor development to quantify the natural history of disease progression in a relatively large number of individuals 
with ALS. The work is currently funded by an initiative from the Vice Chancellors of Research office to facilitate the development of intercampus consortiums that engage members from a wide number of disciplines.

\section{Summary}

In summary, I have given the researcher's perspective on current challenges faced by my discipline and considered several measures to address these challenges which include (1) train the next generation of scientists, (2) do bigger, better science through consortiums, (3) focus funding efforts toward overcoming technological hurdles, and (4) provide continuing education for scientists. A number of outstanding initiatives have already been put into place by the federal government, professional agencies, and universities to address many of these issues. These initiatives, although modest in size, suggest the research community is headed in the right direction.

\section{References}

1. Giles, J. \& Wadman, M. (2006). Grants fall victim to NIH success, Nature, 443, 894-895.

Footnote: One overly simplistic - but nonetheless revealing - demonstration of the paucity of studies on speech physiology is the results from a PubMed database search that was limited to the past five years of research. While the terms "grasp and hand" yielded 1882 publications, "speech and tongue" only yielded 285 .

Acknowledgements: Support for this work was provided by NIH Grant \#5R01DC006463-03 and the Barkley Trust. The author would also like to thank, David Beukelman, Thomas Campbell, Christopher Moore, and Gary Weismer for their insights on topics considered in this paper 